\title{
Influence of the irradiation temperature on the intracascade ion mixing
}

\author{
M. Alurralde, ${ }^{\text {a) }}$ A. Caro, and M. Victoria \\ Paul Scherrer Institute, 5232 Villigen-PSI, Switzerland
}

(Received 26 June 1992; accepted 9 November 1992)

\begin{abstract}
We present a description of the thermal behavior of cascades in $\mathrm{Cu}$ and $\mathrm{Ag}$ over a large energy range and irradiation temperatures. For this purpose the binary collision approximation, which gives the profile of the energy deposition, is coupled to a simplified version of the heat equation. In the present calculations, the original liquid drop model [M. Alurralde, A. Caro, and M. Victoria, J. Nucl. Mater. 183, 33 (1991)] has been extended to the case where the lattice is at finite temperatures. The resulting evolution of the liquid cascade is analyzed for PKA energies up to $1 \mathrm{MeV}$, and the results are compared to experimental observations of mixing rates. We obtain a temperature dependence that adds to the traditional Radiation Enhanced Diffusion, RED, in very good qualitative agreement with experiments on materials showing thermal spikes.
\end{abstract}

\section{INTRODUCTION}

The parameters that are relevant in understanding the microscopic evolution of displacement damage cascades, such as the number of freely migrating defects or the rate of atomic mixing, can be correctly studied using molecular dynamics simulations, MD. Present-day computer capabilities, though, allow MD simulations with energies below approximately $25 \mathrm{keV} .^{1}$

Some of the most important results of MD simulations are the prediction of the cascade core melting and the conclusion that the dominant contribution to ion mixing, IM, at low irradiation temperatures is intracascade mass transport during the cooling phase. ${ }^{1-3}$ The dependence of this effect on lattice temperature for $3 \mathrm{keV}$ cascades in $\mathrm{Cu}$ has been studied in Ref. 4. In a previous investigation we assumed that this melting picture is correct for $\mathrm{Cu}$, and applied simple thermodynamics to predict the behavior of higher energy cascades. 5,6 Previous alternative attempts to solve the cascade evolution using continuum heat equations gave less information because both the energy deposition profile and the thermodynamic factors entering the equation are in fact unknown, forcing considerable simplifications. ${ }^{7,8} \mathrm{By}$ coupling the binary collision approximation, BCA, to a simplified version of the heat equation, we obtained a description of the cascade behavior in several materials over large energy and temperature ranges, correctly accounting for the geometric aspects. ${ }^{5,6}$ This description provided an estimation of subcascade interactions, in an energy range inaccessible to MD with present-day computational performances.

\footnotetext{
a) On leave from the Comisión Nacional de Energía Atómica, Libertador 8250, (1429) Buenos Aires, Argentina.
}

In the present work we address the problem of the role of lattice temperature on the behavior of cascades within the framework of the liquid drop model. Besides the obvious effect of increasing the lifetime of the heat spike, two additional effects are expected. One is the decrease of the length of the replacement collision sequences, RCS, as a consequence of the distortion induced by thermal fluctuations along the compact directions. The other is the decrease of the length of focusons produced by the same fluctuations. Both RCS and focusons are efficient ways to transport energy away from the cascade core, thus decreasing the energy density. RCS appear during the collisional phase, and are therefore well described in the BCA, which includes temperature as random displacement of target atoms around their equilibrium positions. Focusons are neither included in BCA nor in the continuous heat equation; their role will be neglected on the basis of a comparison with molecular dynamics. The increases in lifetime, temperature, and energy density combine to enhance total atomic motion or ion mixing during the cooling phase. The temperature dependence of this effect adds to the traditional radiation enhanced diffusion at intermediate temperatures, RED, which is mass transport due to freely migrating vacancy and interstitial defects.

\section{COMPUTATIONAL METHOD}

The calculations are performed using the Marlowe12 code. ${ }^{9}$ It is based on the binary collision approximation, in which the cascade develops inside a crystalline solid as a sequence of two-body collisions. The crystalline structure is given by the locations of the target atoms. An atom is set into motion if, as a consequence of a collision, it receives an energy larger than some 
cutoff, $E_{c}$. Similarly, an atom no longer moves if its energy falls below $E_{c}$. A cascade ends when all atoms have an energy lower than $E_{c}$. Additional parameters are a binding energy, $E_{b}$, which is lost every time an atom is displaced from its perfect lattice position, and the parameters controlling the inelastic electronic losses. In this work we use internal options of Marlowe for the pair potential, as well as for inelastic losses. They correspond to the Moliere approximation to the Thomas Fermi screening, with a screening length given by matching the Moliere potential at the nearest neighbor distance to a Born-Mayer potential fitted to bulk equilibrium data. Inelastic losses are incorporated in a modified version of the model proposed by Firsov. ${ }^{9}$ The output configuration contains the positions and kinetic energies of all atoms set into motion; it represents the end of the collisional phase of the cascade. In order to associate the spatial distribution of this amount of energy to the real deposited energy density, on which the volume of the melt is strongly dependent, we analyze the energy balance at the end of the collisional phase of the cascade as follows: the total initial energy, $E_{\mathrm{PKA}}$, i.e., the kinetic energy of the primary knock-on atom (PKA), is distributed among (a) the inelastic collisions with electrons, $E_{i}$, (b) the kinetic energy of the atoms that entered the cascade, $E_{k}$, and (c) the binding energy lost at each collision, $E_{b}$ (which represents the potential energy of the distorted lattice and the excitations of vibrations), plus the energy below the cutoff transferred to those atoms never entering the cascade. This last contribution is called damage energy, $E_{d}$. Therefore,

$$
E_{\mathrm{PKA}}=E_{i}+E_{d}+E_{k} .
$$

The output configuration contains the positions and kinetic energies of all atoms set into motion. It represents the end of the collisional phase of the cascade. A simple way to relate the spatial distribution of this amount of energy to the real deposited energy density, since the only available information in the binary collision code is the last location of the moved atoms, is to apply a multiplicative factor, $E_{\mathrm{PKA}} / E_{k}$, to the energy of each of these atoms. In this way the correct total amount of energy is deposited in the crystal but at the last location of the moving atoms, instead of being along their trajectories. As we showed in Refs. 5 and 6 , this approximation does not significantly affect the energy density profile. The energy density at the end of the collisional phase is transformed into heat by thermalization and then propagated.

The equation of heat conduction in an isotropic, uniform medium with thermal conductivity, $\kappa$, specific heat $C$, and density $\rho$ is:

$$
\frac{\partial Q}{\partial t}=\nabla \cdot\{(\kappa / C \rho) \nabla Q\}
$$

$\rho$ and $\kappa$ are functions of $T$, and therefore of $\mathbf{r}$ and $t$. However, comparisons with MD results, justify taking spatial averages of these quantities over the whole volume of integration, \langle\rangle , and defining $a_{0}$ such that,

$$
\Delta t=\left\langle C \rho a_{0}^{2} / 7 \kappa\right\rangle,
$$

we can write a discrete version of Eq. (2) in a simple cubic partitioning of the space in cells of linear dimension $a$,

$$
\begin{aligned}
Q(\mathbf{r}, t+\Delta t)= & \left(a_{0}^{2} / 7 a^{2}\right)\{Q(x+a, y, z, t) \\
& +Q(x-a, y, z, t)+Q(x, y+a, z, t) \\
& +Q(x, y-a, z, t)+Q(x, y, z+a, t) \\
& +Q(x, y, z-a, t) \\
& \left.+\left[7 a^{2} / a_{0}^{2}-6\right] Q(\mathbf{r}, t)\right\} .
\end{aligned}
$$

In this equation we neglect the presence of the liquidsolid interface. Time is then measured in unknown units $\Delta t$ that we call hereafter steps. It should be noted that this unit is not linearly related to time because $\kappa$ changes with temperature. This approximation is discussed in detail in Refs. 5 and 6.

To determine the volume of the melt we follow two procedures. First, we evaluate the energy necessary to heat the volume associated to one atom from $0 \mathrm{~K}$ up to the melting temperature; we call this value $Q_{1}$. In the second procedure, we just add to $Q_{1}$ the latent heat of melting per atom $L$, obtaining $Q_{2}$. The differences between these two values are the changes in the potential energy of the ions and the modifications of the electronic contributions to the total energy in going from the solid to the liquid. Both strongly depend on the material. Because the phase transition in the cascade is far from usual conditions (in particular the contribution from electrons is difficult to quantify), we interpret these two values as giving the lower and upper limits of the true energy required to melt.

To evaluate the thermal spike contribution to ion mixing, we assume, as usual, that a simple thermally activated jump process takes place in the liquid phase. In this approximation we neglect all the effects due to the presence of perturbations and defects in the melt and, what is more important, we neglect the contribution of the thermal gradient to the flux of particles; a better description would be that of a diffusion equation for the particles. Under these assumptions, the total average squared distance, which we call ion mixing, IM, traveled by the diffusing particles, per cascade, is given by,

$$
\operatorname{IM}\left\langle\mathbf{L}(t)^{2}\right\rangle=6 \int d t^{\prime} \int \rho_{\mathrm{at}} D(T) d^{3} \mathbf{r}
$$

where $D(T)$ is the diffusion coefficient given by an Arrhenius law,

$$
D(T)=D_{0} \exp \left(-H_{m} / k T\right)
$$


Table I in Ref. 6 shows the experimental values of the diffusion coefficients in the liquid phase as well as their validity ranges.

Results are reported on a large number of cascades in $\mathrm{Cu}$ and $\mathrm{Ag}$, at $0 \mathrm{~K}, 300 \mathrm{~K}, 700 \mathrm{~K}$, and at $75 \%$ of the melting temperature. The PKA energy is ranged from 10 to $1000 \mathrm{keV}$ in decades. One additional case was run in $\mathrm{Cu}$ at $3 \mathrm{keV}$ in order to compare with the MD results of Ref. 4. The influence of the initial parameters of the PKA (position and direction of motion), has been analyzed in a previous publication. ${ }^{6}$ The results exhibit a standard deviation of only $15 \%$ for the volume and $13 \%$ for the damage energy despite significant differences in shape. The internal Marlowe options were used to define the lattice temperature during the collisional phase.

\section{RESULTS}

As was shown both in MD calculations and in our previous results, ${ }^{6}$ the molten volume starts increasing, reaches a maximum, and then decreases as a function of time, for both melting criteria $Q_{1}$ or $Q_{2}$. This evolution is material dependent, because it depends on the deposited energy density. In order to compare our results with those obtained using MD, a first case was run with a $3 \mathrm{keV}$ PKA in $\mathrm{Cu}$, for which MD results are available. The results are shown in Fig. 1, in which the maximum molten volume is plotted as a function of the lattice temperature, normalized to the melting temperature. In the results of Hsieh et al., ${ }^{4}$ the melting temperature used was $1000 \mathrm{~K}$, as obtained from the Gibson II potential.

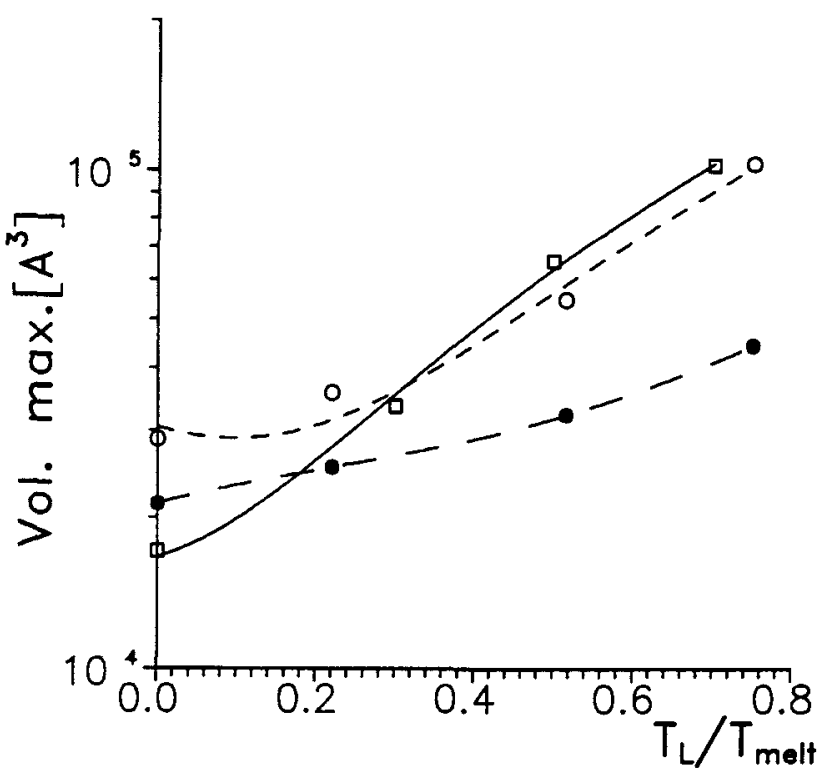

FIG. 1. The maximum molten volume is plotted as a function of the lattice temperature normalized to the melting temperature. ( $\square$ ) Hsieh et al. ${ }^{4} ;(0)$ and $(\bullet)$ considering $Q_{1}$ and $Q_{2}$, respectively. The lines are only indicative.
For our calculations, it was $1356 \mathrm{~K}$. The results using $Q_{1}$ show a good agreement, probably due to the effect of the heat of melting resulting from the use of the Gibson II potential in the MD calculation, which is not reported. According to our experience, the heat of melting predicted by the empirical potentials used in $\mathrm{MD}$ simulations can be as much as a factor of two off the experimental values. As was argued in Ref. 6, since the conditions of the phase transition inside the cascade are far from equilibrium, the results in terms of $Q_{1}$ and $Q_{2}$ should be interpreted as the lower and upper boundaries of the true energy required to melt.

In Fig. 2, the evolution of the ratio between damage energy deposited in replacement collision sequences $\left(E_{\mathrm{RCS}}^{D}\right)$ to total damage energy $\left(E^{D}\right)$, normalized to its value at $0 \mathrm{~K},\left[E_{\mathrm{RCS}}^{D}(T) / E^{D}(T)\right] /\left[E_{\mathrm{RCS}}^{D}(T=0 \mathrm{~K}) /\right.$ $\left.E^{D}(T=0 \mathrm{~K})\right]=Y(T) / Y(0)$, is plotted as a function of the lattice temperature for $100 \mathrm{keV}$ PKA energies $[Y(T=0)=0.56$ in $\mathrm{Ag}$ and 0.43 in $\mathrm{Cu}]$. These curves show that the overall influence of replacement collisions is not significant. In fact, it can be observed that heating the lattice at $1000 \mathrm{~K}$ reduces the energy carried out by RCS by only $10 \%$ in both materials. Therefore the increase in energy density that ensues is small.

The maximum volume of the liquid in $\mathrm{Cu}$ and $\mathrm{Ag}$ as a function of the lattice temperature, for several PKA energies, is shown in Figs. 3(a) and 4(a). The time dependence of the difference between the maximum and the lattice temperatures is plotted in Figs. 3(b) and 4(b). All four lattice temperatures are reported on the same plot, each curve representing a different PKA

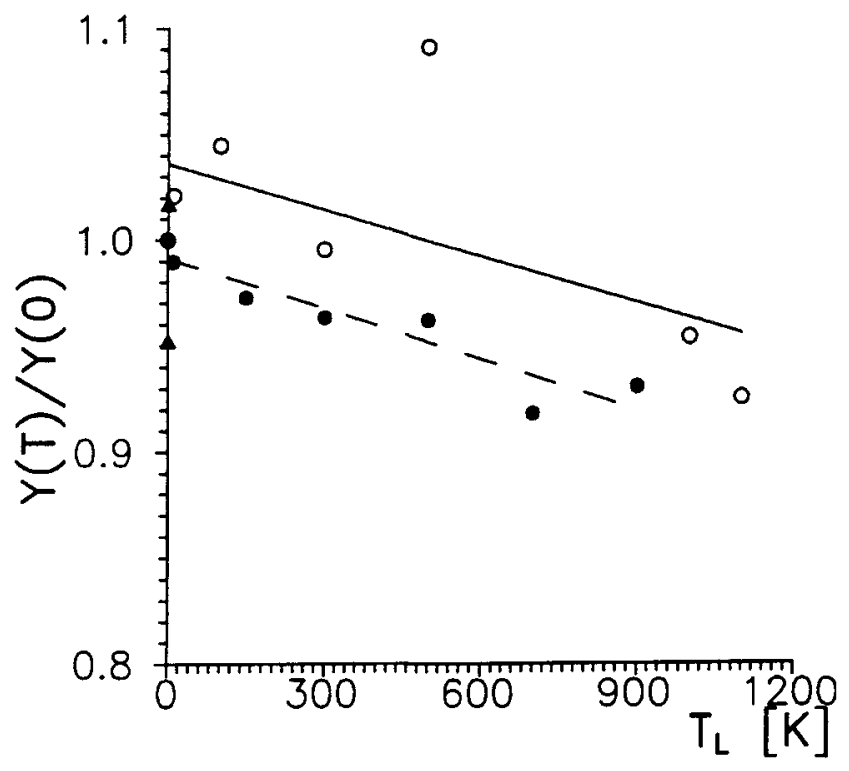

FIG. 2. Ratio of the damage energy deposited in RCS to damage energy, normalized to this ratio at $0 \mathrm{~K}$, plotted as a function of the lattice temperature. The symbols (O) and $(\bullet)$ are for $\mathrm{Cu}$ and $\mathrm{Ag}$, respectively. The lines are only indicative. 


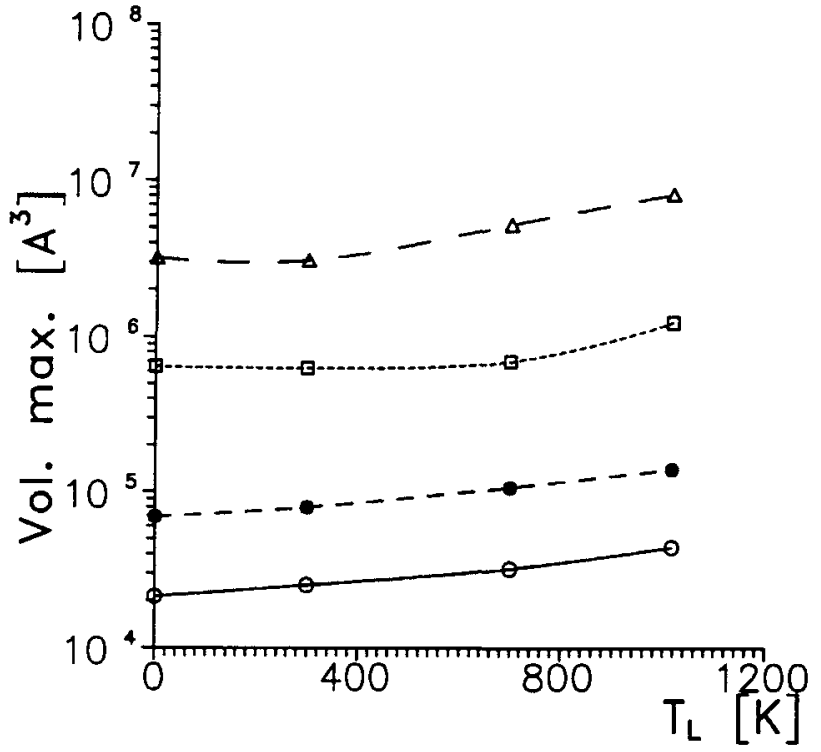

(a)

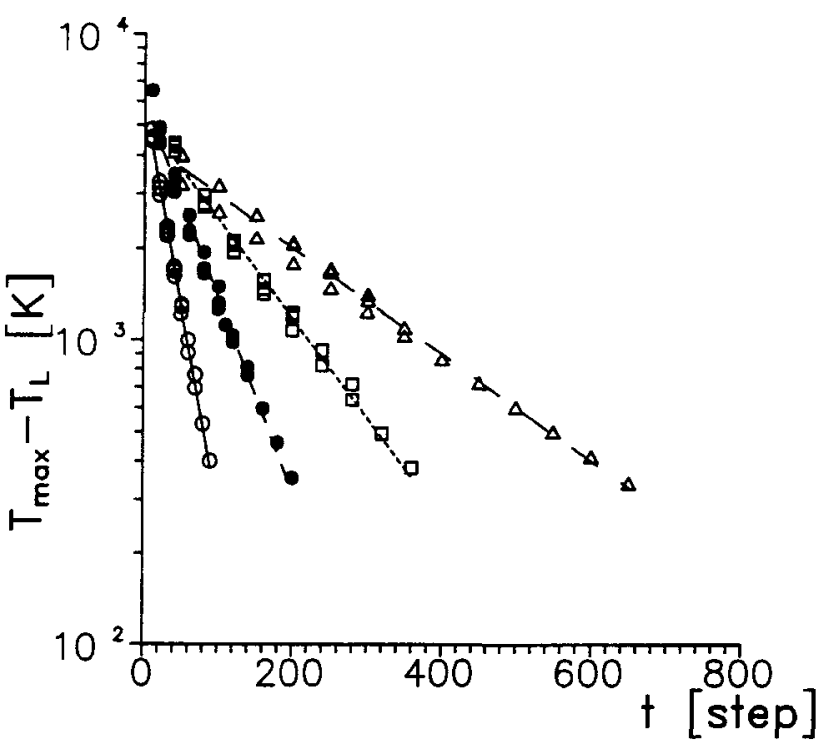

(b)

FIG. 3. (a) The maximum volume of the melt as a function of the lattice temperature for different PKA energies in $\mathrm{Cu}$. (O) $3 \mathrm{keV}$, $(\bullet) 10 \mathrm{keV},(\square) 100 \mathrm{keV}$, and $(\triangle) 1000 \mathrm{keV}$. (b) The time dependence (in discrete steps, see Ref. 6) of the difference between the maximum temperature in the liquid volume and the lattice temperature. The symbols are the same as (a).

energy. The influence of the lattice temperature on the $\mathrm{RCS}$ behavior in the collisional phase is visible in $\mathrm{Ag}$ where, for a given PKA energy and time, this normalized maximum temperature has some dispersion for different lattice temperatures; the vertical splitting of the data points reflects that the higher the lattice temperature is, the higher the energy density results. This effect of RCS is much less pronounced in $\mathrm{Cu}$.

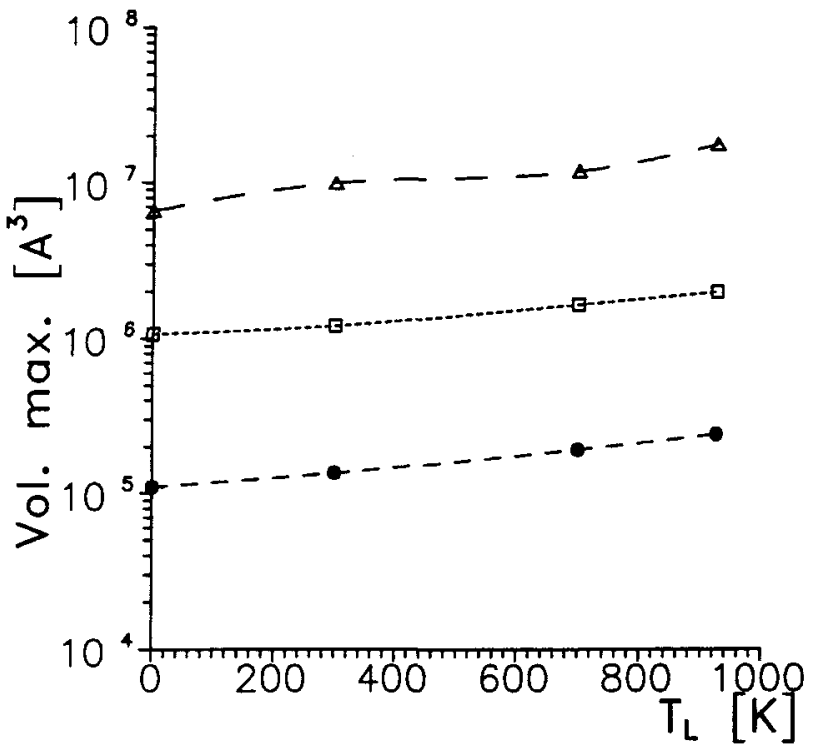

(a)

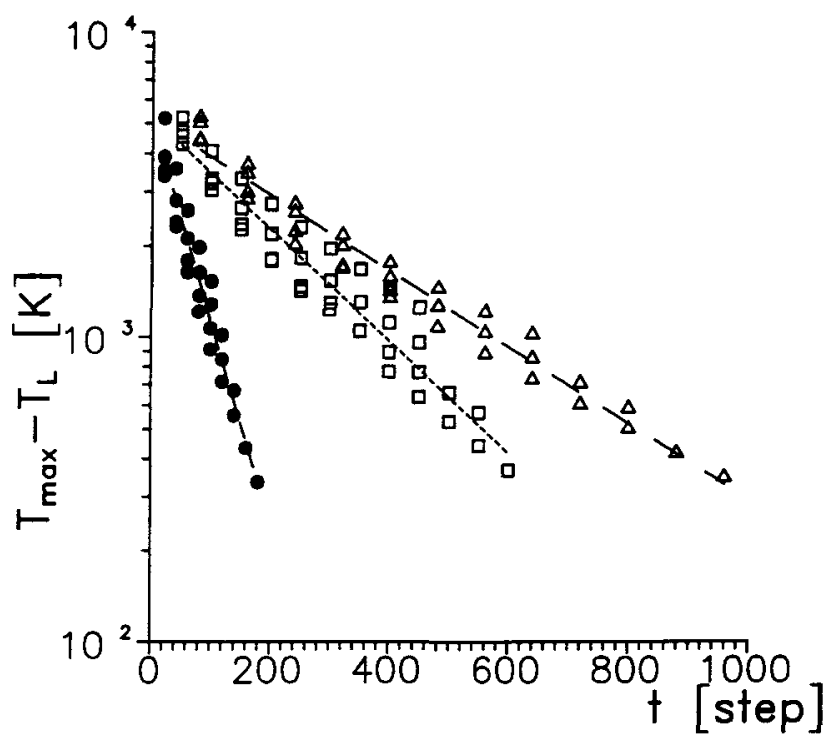

(b)

FIG. 4. (a) The maximum volume of the melt as a function of the lattice temperature for different PKA energies in Ag. (•) $10 \mathrm{keV}$, ( $\square) 100 \mathrm{keV}$, and $(\triangle) 1000 \mathrm{keV}$. (b) The time dependence (in discrete steps, see Ref. 6 ) of the difference between the maximum temperature in the liquid volume and the lattice temperature. The symbols are the same as (a).

The overall time behavior in Figs. 3(b) and 4(b) is a decreasing exponential, as compared to the power law with exponent -1.5 predicted by analytic models, ${ }^{7,8}$ assuming that the initial profile of the energy deposition is delta-type or Gaussian. The data obtained in the present calculation refer to the short time behavior (liquid phase) and suggest that the early temperature distribution is not Gaussian; at longer times the distribution probably 
becomes Gaussian, recovering the power law evolution, as observed by Hsieh et al. ${ }^{4}$ in MD.

\section{DISCUSSION}

A different way of presenting the results is the Arrhenius plots in Figs. 5(a) and 5(b). These curves are typical of ion mixing, and when they also include the high temperature thermally activated diffusion, they are known as " $Q$-curves". Three temperature regimes are usually associated with them. The low temperature, where only a weak dependence of mixing on irradiation temperature is found, is the consequence of ballistic mixing during the collisional phase; intermediate temperatures, typically beginning near or slightly above room temperature, where a temperature dependence starts to develop, are the regime of Radiation Enhanced Diffusion (RED) where the excess vacancy and interstitial defects produced during irradiation are supposed to become sufficiently mobile to enhance mass transport; and a high temperature regime, where the rate of thermally induced interdiffusion is the controlling mechanism, while irradiation plays a minor role. ${ }^{10}$

These curves have been the subject of intensive study, both theoretical and experimental for many years. For some materials, like Ni single-crystals, the RED regime is clearly understood; the apparent activation enthalpy is one-half of the vacancy migration enthalpy, as expected. For other systems, like $\mathrm{Nb} / \mathrm{Si}$ and U-based bilayers, the apparent activation enthalpy depends on the ion species, which is clearly inconsistent with the RED assumptions. ${ }^{11,12}$ Additional observations on amorphous $\mathrm{Si}$, where strong temperature but no dose-rate dependences are reported, ${ }^{13}$ or in amorphous $\mathrm{Ni} / \mathrm{Zr}$ where a pronounced dose-rate dependence appears, ${ }^{14}$ suggest that intracascade as well as intercascade processes are relevant to the enhanced mixing at intermediate temperatures.

In fact, the picture that emerges from these observations and from MD simulations is that the majority of the transport occurs inside the thermal spike and is subjected to dependence on the usual thermodynamic variables like heat of mixing or cohesive energy. Strong support for this explanation was reported on Cu bilayers which form miscible liquids but have low solubility in the solid phase. ${ }^{15}$

Modeling of this effect using thermodynamic mixing has recently been applied to observations on U bilayers. ${ }^{16}$ Assuming an empirical expression for the spike size dependence on irradiation temperature, a typical " $Q$ curve" is obtained whose apparent activation enthalpy is clearly not related to RED. Also reported is the significant influence of the subcascade overlap on the mixing rate.

From the above discussion, it appears that the intermediate temperature regime of ion mixing can contain

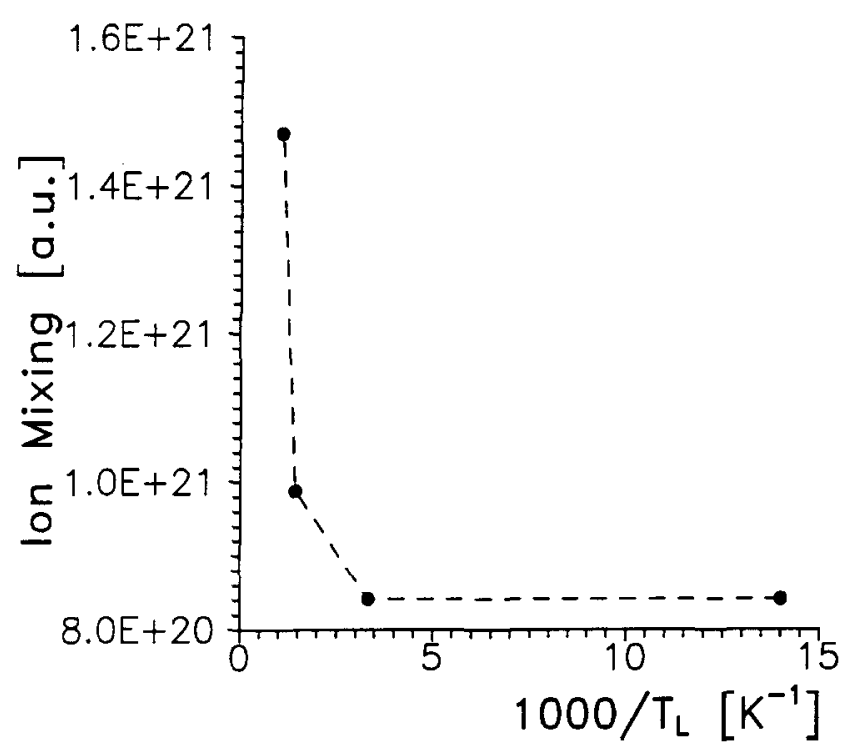

(a)

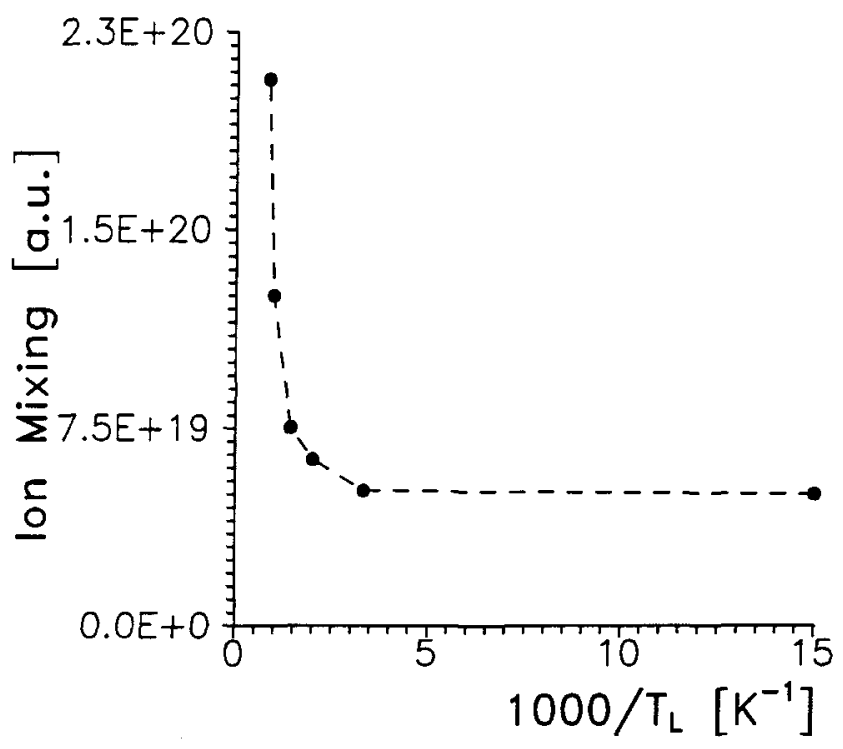

(b)

FIG. 5. Total ion mixing per cascade in arbitrary units (a.u.), Eq. (5), as a function of the inverse of irradiation temperature. (a) $\mathrm{Cu}, 100 \mathrm{keV}$ and (b) $\mathrm{Ag}, 1 \mathrm{MeV}$.

a significant contribution from intra- and intercascade mixing, which adds to traditional RED in those systems showing hot spikes. It becomes then an ideal field to apply the liquid drop model of ion mixing. ${ }^{5,6}$ The results shown in Figs. 5(a) and 5(b) indicate that the intermediate temperature regime starts around room temperature in both $\mathrm{Cu}$ and $\mathrm{Ag}$ and since the temperature dependence is a consequence of the combined effects of increase of spike temperature and lifetime, an apparent activation energy cannot be associated with the process. The magnitude of this effect is comparable to the experimental observations reported for U-based bilayers (see Fig. 8 
in Ref. 12), and is much less than the well-behaved $\mathrm{Ni}$ RED results (see Fig. 3 in Ref. 10), where the spikes are known to be shorter.

In conclusion, we have shown, using computer simulations, the influence of the irradiation temperature in the intracascade ion mixing in such a way that a correct description of the geometrical aspects of the collision process, as well as the subcascade interactions, were incorporated. The results show a very good agreement with experimental observations.

\section{REFERENCES}

1. T. Diaz de la Rubia and M. Guinan, Phys. Rev. Lett. 66, 2766 (1991).

2. T. Diaz de la Rubia, R. S. Averback, R. Benedek, and W. E. King, Phys. Rev. Lett. 59, 1930 (1987).

3. T. Diaz de la Rubia, R.S. Averback, H. Hsieh, and R. Benedek, J. Mater. Res. 4, 579 (1989).

4. H. Hsieh, T. Diaz de la Rubia, R.S. Averback, and R. Benedek, Phys. Rev. B 40, 9986 (1989).

5. M. Caro, A. Ardelea, and A. Caro, J. Mater. Res. 5, 2652 (1990).

6. M. Alurralde, A. Caro, and M. Victoria, J. Nucl. Mater. 183, 33 (1991).
7. G. H. Vineyard, Radiat. Eff. 29, 245 (1976).

8. J. B. Sanders, Radiat. Eff. 51, 43 (1980); D. Peak and R. S. Averback, Nucl. Instrum. Methods B 7/8, 561 (1985).

9. M. T. Robinson and I. M. Torrens, Phys. Rev. B 9, 5008 (1974).

10. L.E. Rehn and P. R. Okamoto, Nucl. Instrum. Methods B 39, 104 (1989).

11. U. Shreter, F. C. T. So, B. M. Paine, and M. A. Nicolet, in Ion Implantation and Ion Beam Processing of Materials, edited by G. K. Hubler, O. W. Holland, C. R. Clayton, and C. W. White (Mater. Res. Soc. Symp. Proc. 27, Elsevier Science Publishing, New York, 1984), p. 31.

12. F. Rossi, M. Nastasi, M. Cohen, C. Olsen, J. R. Tesmer, and C. Egert, J. Mater. Res. 6, 1175 (1991).

13. F. Priolo, J. M. Poate, D. C. Jacobson, J. Linnros, J. L. Batstone, and S. U. Campisano, in Fundamentals of Beam-Solid Interactions and Transient Thermal Processing, edited by M.J. Aziz, L.E. Rehn, and B. Stritzker (Mater. Res. Soc. Symp. Proc. 100, Pittsburgh, PA, 1988), p. 87.

14. F. R. Ding, R. S. Averback, and H. Hahn, J. Appl. Phys. 64, 1785 (1988).

15. R. S. Averback, D. Peak, and L. J. Thompson, Appl. Phys. A 39, 59 (1986).

16. W. L. Johnson, Y. T. Chen, M. van Rossum, and M. A. Nicolet, Nucl. Instrum. Methods B 7/8, 657 (1985). 